\title{
Determinants of utilization of antenatal and postnatal care services in Telangana
}

\author{
Neelima Singh ${ }^{1}$, Satyendra Nath Ponna ${ }^{1 *}$, Venkata Prasad Upadrasta ${ }^{2}$, \\ Shankar Reddy Dudala ${ }^{3}$, Renuka Sadasivuni
}

\author{
${ }^{1}$ Indian Institute of Health and Family Welfare, Hyderabad, Telangana, India \\ ${ }^{2}$ MRHRU, Chandragiri, Chittoor, Andhra Pradesh, India \\ ${ }^{3}$ SV Medical College, Tirupati, Andhra Pradesh, India
}

Received: 13 July 2017

Accepted: 15 July 2017

\section{*Correspondence:}

Dr. Satyendra Nath Ponna,

E-mail: snponna@gmail.com

Copyright: () the author(s), publisher and licensee Medip Academy. This is an open-access article distributed under the terms of the Creative Commons Attribution Non-Commercial License, which permits unrestricted non-commercial use, distribution, and reproduction in any medium, provided the original work is properly cited.

\section{ABSTRACT}

Background: Variation exists in utilization of antenatal and postnatal care services in public health facilities of developing countries. Provision of antenatal and postnatal care services is the major function of public health delivery system of India to improve maternal health outcomes. The objective of this study is to estimate the determinants of utilization of antenatal and postnatal care services stratified by geographical region in Telangana.

Methods: It is a cross-sectional study of District Level Health and Facility Survey-4 of the state of Telangana. Multistage, stratified, probability proportional to size sample with replacement was used. 3065 women, who delivered after the year 2008, were considered for analysis. Descriptive analysis of components of antenatal and postnatal care services stratified by geographical region was carried out. Binomial logistic regression was carried out to determine association of demographic, system level variables with adequate antenatal care.

Results: Study reveals variation exists across four regions of Telangana in utilization of maternal health services. Reception of adequate antenatal care is low in South region (20.6\%) and high in East region (31.5\%). Pregnant women with secondary education were $66 \%$ more likely to receive adequate antenatal care services compared to illiterate.

Conclusions: Short term and long-term goals to be adopted and implemented by government to address the demandsupply imbalance such as public health infrastructure and quality of services in underperforming districts of Telangana to increase utilization of maternal health services by training health staff and engaging local communities to seek health care services.

Keywords: Antenatal care, Determinants, District level house hold facility survey, Geographical region, Postnatal care, Telangana

\section{INTRODUCTION}

In developing countries, there is wide gap between rural and urban communities and across geographical regions, in utilization of maternal health services. ${ }^{1}$ Also, United Nations (UN) Sustainable Development Goals (SDG) mandate of 2015 highlights to reduce maternal and infant mortality rates. ${ }^{2}$ Universal health care aimed at ensuring equitable access for all Indian citizens, resident in any part of the country, regardless of income level, social status, gender, caste or religion, to affordable, accountable, appropriate health services of assured quality. ${ }^{3}$ Despite these efforts, moderate penetration of health services in rural settlements were still not able to access these services and the evidence is clear. ${ }^{4}$ Evidence 
from previous studies reveal one fourth of the mothers in the year 2004-2005 were unable to use services because of cost of health services. ${ }^{5}$ Disparities exist in the utilization of health services between urban and rural populations; and inequities exist in availability and accessibility of maternal health care services. ${ }^{6}$

In India, the studies show utilization of the maternal health services by urban and affluent population was higher than rural and poor. ${ }^{6,7}$ The National Family Health Survey-3 data show that the utilization of antenatal care (ANC) and medical assistance (the odds of ANC being 1.48 , medical assistance in delivery 2.16) in wealthiest quintiles than the poor in urban areas. ${ }^{8}$ In India, maternal health services are free in public health centres but out of pocket health expenditure is two thirds of the total expenditure and health care costs are catastrophic., ${ }^{9,10}$ WHO recommends that every pregnant woman should have undergone at least four goal-oriented focused ANC visits under the supervision of skilled provider and should be commenced as early as possible in first trimester which includes all interventions on regular intervals throughout the pregnancy. ${ }^{11}$

There is a strong correlation between adequate ANC and the maternal health and regular ANC gives opportunity to monitor and correct adverse health outcomes of the maternal related health issues. ${ }^{12,13}$ As per the WHO randomised trial on ANC four antenatal visits are essential to monitor for mother and foetus wellbeing. ${ }^{14,15}$ It is also reported that women with poor socio-economic background, low levels of education had low utilization of the antenatal services. ${ }^{16-18}$

To identify the barriers to access of utilizing antenatal services, data is required. The present study aims to assess the health care needs, utilization and to identify demand side barriers with regard to the ANC services and post-natal care services in the state of Telangana.

\section{METHODS}

\section{Study location}

The state of Telangana emerged as $29^{\text {th }}$ state of union of India on June $2^{\text {nd }}, 2014$ and is the $12^{\text {th }}$ largest state in India with an area of 1,12,077 square kilometres and population of $350,03,674$ according to 2011 census. The decadal growth rate of population of Telangana is in declining phase, $13.58 \%$ in $2001-2011$ and $18.77 \%$ in 1991-2001 and is lower than national average (17.64\%). Population density of state ranges from 170 to 18172 per square kilometre, with lowest in district of Adilabad and highest in Hyderabad. Majority of population resides in rural areas with $38.9 \%$ residing in urban areas. The state had $9.04 \%$ and $15.45 \%$ of scheduled tribe and scheduled caste population. The per-capita income of the state of Telangana is 1.29 lac in the year of $2014-15$, but it is skewed with highest per capita income in Hyderabad $(2,94,200)$ and lowest in Adilabad $(76,921) .{ }^{19}$
The state comprises 10 administrative districts, 459 mandals and 9834 villages. There is a wide geographic, economic and social disparity among districts. Human Development Index (HDI) of Telangana is 0.513 in 201112, while district of Hyderabad and Medak has HDI of 0.764 and 0.446 , respectively. The accountability of provision of maternal health services in the state is under the department of Public Health and Family Welfare of state government and Telangana Vaidya Vidhana Parishad (TVVP). These services are catered through 108 general hospitals and 671 primary health centres. The state also has large network of private hospitals densely distributed in urban settlements.

\section{Study design and sampling}

District Level Household and Facility Survey (DLHS-4) was a fourth round of nationwide survey conducted in 2012-13. It is a multi-stage, stratified, probability proportional to size (PPS) sample with replacement, was adopted in 21 states of DLHS-4 (Except Gujarat).

The First Stage Unit (FSU) for urban areas is the National Sample Survey Organization (NSSO), Urban Frame Survey (UFS) blocks and Ultimate Stage Sampling Unit (USU) was the household (HH). UFS blocks in each district were stratified into million class cities and nonmillion class cities and allotment of sample was proportional to relative sizes. Sample frame for selection of Primary Sampling Units (PSU) for urban areas was provided by Survey Design and Research Division (SDRD) of NSSO. Urban areas in some districts were over sampled by taking more PSUs for districts with less than estimated $30 \%$ urban population. Household number per PSU is 25. While for rural areas, census 2001 villages were framed as FSU of sampling, stratified by size and selection by PPS. Households listing in the FSU provided the sampling frame for selecting households at the second stage.

List of household preparation served as the sampling frame for selection of representative households. This involved mapping and listing of structures and households for each sampled PSU following the preparation of location and lay out maps. Large sample villages (more than 300 households) were segmented. For villages with 300-600 HHs, the study had two segments almost with same size and one was selected randomly among them. For villages with more than $600 \mathrm{HHs}$, more than three segments were made with about 150 households each and two were selected at random. In urban areas, each UFS have no more than 300 HHs. Each $\mathrm{HH}$ district had one lister and one mapper, this operation was completed in two months in advance of the survey. PSU were selected in each district from rural and urban sample frame followed by selection of households. Allocation of HHs is proportional to size of each substratum. Each selected rural/urban PSU is represented by $25 \mathrm{HH}$. 
Table 1: Socio-demographic variation in each region of Telangana.

\begin{tabular}{|c|c|c|c|c|}
\hline \multirow[b]{2}{*}{ Socio-Demographic Variables } & \multicolumn{4}{|l|}{ Regions } \\
\hline & $\begin{array}{l}\text { North\# } \\
(\mathbf{n}=\mathbf{8 0 5})\end{array}$ & $\begin{array}{l}\text { East\# } \\
(n=753)\end{array}$ & $\begin{array}{l}\text { South\# } \\
(n=591)\end{array}$ & $\begin{array}{l}\text { West\# } \\
(n=916)\end{array}$ \\
\hline \multicolumn{5}{|l|}{ Mother's age (years) } \\
\hline$\leq 20$ & $7.8(6.0,9.7)$ & $9.8(7.7,12.0)$ & $11.0(8.5,13.5)$ & $8.6(6.8,10.4)$ \\
\hline 21 to 25 & $44.6(41.2,48.0)$ & $42.8(39.2,46.3)$ & $42.6(38.7,46.6)$ & $44.8(41.5,48.0)$ \\
\hline 26 to 30 & $34.3(31.0,37.6)$ & $36.0(32.6,39.4)$ & $33.8(30.0,37.7)$ & $33.7(30.7,36.8)$ \\
\hline$\geq 31$ & $13.3(10.9,15.6)$ & $11.4(9.1,13.7)$ & $12.5(9.9,15.2)$ & $12.9(10.7,15.1)$ \\
\hline \multicolumn{5}{|l|}{ Age at marriage (years) } \\
\hline$<18$ & $24.2(21.3,27.2)$ & $24.4(21.4,27.5)$ & $29.6(25.9,33.3)$ & $28.9(26.0,31.9)$ \\
\hline 18 to 20 & $55.7(52.2,59.1)$ & $52.7(49.2,56.3)$ & $51.6(47.6,55.6)$ & $51.0(47.7,54.2)$ \\
\hline$\geq 21$ & $20.1(17.4,22.9)$ & $22.8(19.8,25.8)$ & $18.8(15.6,21.9)$ & $20.1(17.5,22.7)$ \\
\hline \multicolumn{5}{|l|}{ Age when first child born (years) } \\
\hline$<21$ & $45.2(41.8,48.7)$ & $49.5(46.0,53.1)$ & $53.6(49.6,57.7)$ & $50.8(47.5,54.0)$ \\
\hline$\geq 21$ & $54.8(51.3,58.2)$ & $50.5(46.9,54.0)$ & $46.4(42.3,50.4)$ & $49.2(46.0,52.5)$ \\
\hline \multicolumn{5}{|l|}{ Religion } \\
\hline Hindu & $85.8(83.4,88.2)$ & $87.4(85.0,89.8)$ & $85.4(82.6,88.3)$ & $79.5(76.9,82.1)$ \\
\hline Muslim & $13.2(10.8,15.5)$ & $8.1(6.2,10.0)$ & $12.5(9.9,15.2)$ & $16.2(13.8,18.5)$ \\
\hline Christian & $1.0(0.3,1.7)$ & $4.5(3.0,6.0)$ & $2.0(0.9,3.2)$ & $4.4(3.0,5.7)$ \\
\hline \multicolumn{5}{|l|}{ Social group } \\
\hline $\mathrm{SC}$ & $34.4(31.1,37.7)$ & $27.0(23.8,30.1)$ & $21.8(18.5,25.2)$ & $25.2(22.4,28.0)$ \\
\hline ST & $13.3(10.9,15.6)$ & $7.8(5.9,9.8)$ & $9.5(7.1,11.8)$ & $3.1(1.9,4.2)$ \\
\hline OBC & $45.8(42.4,49.3)$ & $56.6(53.0,60.1)$ & $59.7(55.8,63.7)$ & $59.6(56.4,62.8)$ \\
\hline General & $6.5(4.8,8.2)$ & $8.6(6.6,10.6)$ & $9.0(6.7,11.3)$ & $12.1(10.0,14.2)$ \\
\hline \multicolumn{5}{|l|}{ Educational status } \\
\hline Illiterate & $38.1(34.8,41.5)$ & $24.6(21.5,27.6)$ & $35.4(31.5,39.2)$ & $31.8(28.8,34.8)$ \\
\hline Primary & $7.2(5.4,9.0)$ & $7.0(5.2,8.9)$ & $4.7(3.0,6.5)$ & $8.8(7.0,10.7)$ \\
\hline Secondary and above & $42.9(39.4,46.3)$ & $50.9(47.3,54.4)$ & $44.0(40.0,48.0)$ & $44.3(41.1,47.5)$ \\
\hline Graduate and above & $11.8(9.6,14.0)$ & $17.5(14.8,20.2)$ & $15.9(13.0,18.9)$ & $15.1(12.7,17.4)$ \\
\hline \multicolumn{5}{|l|}{ Occupational status } \\
\hline Working women & $33.0(29.8,36.3)$ & $23.5(20.5,26.5)$ & $30.3(26.6,34.0)$ & $26.3(23.5,29.2)$ \\
\hline House wife / Student & $67.0(63.7,70.2)$ & $76.5(73.5,79.5)$ & $69.7(66.0,73.4)$ & $73.7(70.8,76.5)$ \\
\hline \multicolumn{5}{|l|}{ Family size } \\
\hline$<5$ members & $35.5(32.2,38.8)$ & $41.3(37.8,44.8)$ & $36.2(32.3,40.1)$ & $37.0(33.9,40.1)$ \\
\hline$\geq 5$ members & $64.5(61.2,67.8)$ & $58.7(55.2,62.2)$ & $63.8(59.9,67.7)$ & $63.0(59.9,66.1)$ \\
\hline \multicolumn{5}{|l|}{ Wealth quintile } \\
\hline Lower Class & $8.2(6.3,10.1)$ & $11.4(9.1,13.7)$ & $15.4(12.5,18.3)$ & $14.3(12.0,16.6)$ \\
\hline Lower Middle Class & $20.7(17.9,23.5)$ & $26.6(23.4,29.7)$ & $25.5(22.0,29.1)$ & $27.0(24.1,29.8)$ \\
\hline Middle Class & $20.7(17.9,23.5)$ & $29.6(26.4,32.9)$ & $28.8(25.1,32.4)$ & $24.8(22.0,27.6)$ \\
\hline Upper Middle Class & $28.2(25.1,31.3)$ & $18.5(15.7,21.2)$ & $18.1(15.0,21.2)$ & $19.9(17.3,22.5)$ \\
\hline Upper Class & $22.1(19.2,25.0)$ & $13.9(11.5,16.4)$ & $12.2(9.5,14.8)$ & $14.1(11.8,16.3)$ \\
\hline \multicolumn{5}{|l|}{ Parity } \\
\hline Primipara & $36.6(33.3,40.0)$ & $39.8(36.3,43.3)$ & $35.0(31.2,38.9)$ & $34.3(31.2,37.4)$ \\
\hline Multipara & $63.4(60.1,66.7)$ & $60.2(56.7,63.7)$ & $65.0(61.2,68.8)$ & $65.7(62.6,68.8)$ \\
\hline \multicolumn{5}{|l|}{ Location } \\
\hline Rural & $61.4(58.0,64.7)$ & $49.4(45.8,53.0)$ & $46.5(42.5,50.6)$ & $40.1(36.9,43.2)$ \\
\hline Urban & $38.6(35.3,42.0)$ & $50.6(47.0,54.2)$ & $53.5(49.4,57.5)$ & $59.9(56.8,63.1)$ \\
\hline
\end{tabular}

Values are proportions.95\% CI in parenthesis. \# North = Adilabad, Nizamabad; East = Karimnagar, Warangal,Khammam; South =

Mahabubnagar, Nalgonda; West $=$ Medak, Hyderabad, Rangareddy.

\section{Data collection}

Computer Assisted Personal Interview (CAPI) was used for data collection in DLHS-4. Each investigator was provided with a mini laptop with bilingual questionnaire.
This technique took minimum time for moving the questionnaire for editing the data and entry etc., from field to work area. CAPI data was uploaded to server located in IIPS (FTP account) day to day base. Bilingual questionnaires were collected in the local language and 
English from Households, visitors stayed in household during the night before interview and ever Married Women in DLHS-4. Standardized HH survey instrument was used to collect information on main source of drinking water, toilet facility, type of fuel used for cooking, caste and religion, education, morbidity and mortality details, multiple marriages, socio-economic status, and assets possessed since January 2008, etc. were collected. Clinical, Anthropometric and Biochemical (CAB) tests were also carried out for selected households.

Table 2: Components of ANC against each region of Telangana.

\begin{tabular}{|c|c|c|c|c|c|}
\hline \multirow[b]{2}{*}{ Components of ANC } & \multicolumn{4}{|l|}{ Regions } & \multirow[b]{2}{*}{$\mathrm{X}^{2}$ (P-value) } \\
\hline & $\begin{array}{l}\text { North }^{\#} \\
(n=805)\end{array}$ & $\begin{array}{l}\text { East }^{\#} \\
(n=753)\end{array}$ & $\begin{array}{l}\text { South }^{\#} \\
(n=591)\end{array}$ & $\begin{array}{l}\text { West }^{\#} \\
(n=916)\end{array}$ & \\
\hline \multicolumn{5}{|l|}{ ANC } & \multirow{3}{*}{$65.7(0.000)$} \\
\hline Inadequate care & $65.2(61.9,68.5)$ & $53.3(49.7,56.8)$ & $60.9(57.0,64.8)$ & $72.1(69.1,75.0)$ & \\
\hline Adequate care* & $34.8(31.5,38.1)$ & $46.7(43.2,50.3)$ & $39.1(35.2,43.0)$ & $27.9(25.0,30.9)$ & \\
\hline \multicolumn{5}{|l|}{ Main Source of ANC } & \multirow{4}{*}{$9.3(0.157)$} \\
\hline Not registered & $4.3(2.9,5.8)$ & $3.6(2.3,4.9)$ & $4.6(2.9,6.3)$ & $3.3(2.1,4.4)$ & \\
\hline Govt. Hospital & $31.9(28.7,35.1)$ & $27.6(24.4,30.8)$ & $32.3(28.5,36.1)$ & $28.5(25.6,31.4)$ & \\
\hline Pvt. Hospital & $63.7(60.4,67.0)$ & $68.8(65.5,72.1)$ & $63.1(59.2,67.0)$ & $68.2(65.2,71.2)$ & \\
\hline \multicolumn{5}{|l|}{ Pregnancy registration } & \multirow{5}{*}{$50.9(0.000)$} \\
\hline Not registered & $4.3(2.9,5.8)$ & $3.6(2.3,4.9)$ & $4.6(2.9,6.3)$ & $3.3(2.1,4.4)$ & \\
\hline 1st trimester & $74.5(71.5,77.5)$ & $80.3(77.5,83.2)$ & $81.7(78.6,84.8)$ & $75.5(72.8,78.3)$ & \\
\hline 2nd trimester & $15.2(12.7,17.6)$ & $13.3(10.9,15.7)$ & $12.5(9.9,15.2)$ & $19.1(16.6,21.7)$ & \\
\hline 3rd trimester & $6.0(4.3,7.6)$ & $2.8(1.6,4.0)$ & $1.2(0.3,2.1)$ & $2.1(1.2,3.0)$ & \\
\hline \multicolumn{5}{|c|}{ Received other ANC services } & \\
\hline Weight & $89.6(87.5,91.7)$ & $94.7(93.1,96.3)$ & $87.3(84.6,90.0)$ & $93.1(91.5,94.8)$ & $30.0(0.000)$ \\
\hline Height & $69.1(65.9,72.3)$ & $74.0(70.8,77.1)$ & $66.8(63.0,70.6)$ & $71.0(68.0,73.9)$ & $9.0(0.029)$ \\
\hline Blood pressure & $82.1(79.5,84.8)$ & $83.0(80.3,85.7)$ & $80.9(77.7,84.1)$ & $78.4(75.7,81.0)$ & $6.7(0.083)$ \\
\hline Blood test $(\mathrm{Hb})$ & $82.0(79.3,84.6)$ & $81.1(78.3,83.9)$ & $82.9(79.9,85.9)$ & $81.3(78.8,83.9)$ & $0.9(0.836)$ \\
\hline Blood test (Blood group) & $78.1(75.3,81.0)$ & $77.2(74.2,80.2)$ & $77.7(74.3,81.0)$ & $70.3(67.3,73.3)$ & $18.9(0.000)$ \\
\hline Blood test (Other) & $73.0(70.0,76.1)$ & $67.1(63.7,70.4)$ & $72.3(68.6,75.9)$ & $61.9(58.8,65.0)$ & $30.3(0.000)$ \\
\hline Urine test & $80.4(77.6,83.1)$ & $80.6(77.8,83.4)$ & $79.5(76.3,82.8)$ & $77.5(74.8,80.2)$ & $3.1(0.370)$ \\
\hline Abdomen examination & $69.6(66.4,72.7)$ & $66.4(63.0,69.8)$ & $67.0(63.2,70.8)$ & $60.3(57.1,63.4)$ & $17.9(0.000)$ \\
\hline Breast examination & $50.9(47.5,54.4)$ & $45.3(41.7,48.8)$ & $50.8(46.7,54.8)$ & $48.1(44.9,51.4)$ & $6.2(0.101)$ \\
\hline Sonogram or Ultrasound & $72.4(69.3,75.5)$ & $74.6(71.5,77.7)$ & $78.5(75.2,81.8)$ & $74.2(71.4,77.1)$ & $6.8(0.077)$ \\
\hline Other test & $4.8(3.4,6.3)$ & $2.4(1.3,3.5)$ & $6.4(4.5,8.4)$ & $3.1(1.9,4.2)$ & $17.7(0.001)$ \\
\hline \multicolumn{5}{|l|}{ IFA consumption } & \multirow{3}{*}{$65.3(0.000)$} \\
\hline Less than 100 days & $57.9(54.5,61.3)$ & $46.9(43.3,50.4)$ & $49.1(45.0,53.1)$ & $64.6(61.5,67.7)$ & \\
\hline Atleast 100 days & $42.1(38.7,45.5)$ & $53.1(49.6,56.7)$ & $50.9(46.9,55.0)$ & $35.4(32.3,38.5)$ & \\
\hline \multicolumn{5}{|l|}{ TT injection } & \multirow{5}{*}{$69.1(0.000)$} \\
\hline Not received & $23.9(20.9,26.8)$ & $11.3(9.0,13.5)$ & $19.3(16.1,22.5)$ & $24.0(21.3,26.8)$ & \\
\hline 1 time & $6.3(4.7,8.0)$ & $8.9(6.9,10.9)$ & $8.1(5.9,10.3)$ & $7.6(5.9,9.4)$ & \\
\hline 2 times & $55.4(52.0,58.8)$ & $63.2(59.8,66.7)$ & $60.1(56.1,64.0)$ & $50.0(46.8,53.2)$ & \\
\hline$>2$ times & $14.4(12.0,16.8)$ & $16.6(13.9,19.3)$ & $12.5(9.9,15.2)$ & $18.3(15.8,20.8)$ & \\
\hline
\end{tabular}

Values are proportions.95\% CI in parenthesis. *ANC adequate care is defined with at least four antenatal visits, at least one tetanus toxoid (TT) injection and took iron folic acid tablets or syrup for 100 or more days. \# North = Adilabad, Nizamabad; East = Karimnagar, Warangal, Khammam; South = Mahabubnagar, Nalgonda; West = Medak, Hyderabad, Rangareddy

Ever-married women's questionnaire was used to collect information from women of 15-49 years on women's characteristics, age, educational status, marriage, pregnancy, mode of delivery including history of induced, spontaneous abortions and still births, antenatal, natal and postnatal care, institutional delivery, financial assistance from Janani Suraksha Yojana (JSY) or any other government schemes, nature of complications during pregnancy for recent births and birth order, postnatal care received by mothers and new born, immunization and childcare, contraception and fertility preferences, reproductive health including knowledge about HIV/AIDS.

DLHS-4survey was conducted in the state of Telangana from 2012 to 2013. Information was collected from 13,927 households, of which 6,918 from rural, and 7,009 from urban regions. 12,432 of total ever married women were surveyed (6,090 from rural and 6,342 from urban). Ever married women, 3,065 HHs (1,508 from rural and 1,557 from urban) delivered after year 2007 was considered for analysis. 
Table 3: Adequate and inadequate ANC against the determinants of ANC.

\begin{tabular}{|c|c|c|c|c|}
\hline & \multicolumn{2}{|l|}{ ANC } & \multirow{2}{*}{$\begin{array}{l}\text { Unadjusted odds ratio } \\
\text { (95\% CI for adequate } \\
\text { versus inadequate) }\end{array}$} & \multirow{2}{*}{$\begin{array}{l}\text { Adjusted odds ratio } \\
\text { (95\% CI for adequate } \\
\text { versus inadequate) }\end{array}$} \\
\hline & $\begin{array}{l}\text { Inadequate care } \\
(n=1946)\end{array}$ & $\begin{array}{l}\text { Adequate } \text { care }^{\wedge} \\
(n=1119)\end{array}$ & & \\
\hline \multicolumn{4}{|l|}{ Mother's age (years) } & \multirow[t]{5}{*}{ 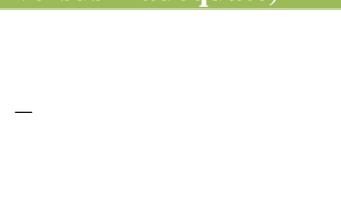 } \\
\hline$\leq 20$ & $9.3(8.0,10.6)$ & $8.9(7.3,10.6)$ & Reference* & \\
\hline 21 to 25 & $45.5(43.3,47.7)$ & $40.9(38.0,43.8)$ & $0.937(0.716,1.226)$ & \\
\hline 26 to 30 & $33.3(31.2,35.4)$ & $36.5(33.6,39.3)$ & $1.140(0.867,1.499)$ & \\
\hline$\geq 31$ & $11.9(10.5,13.4)$ & $13.7(11.7,15.7)$ & $1.194(0.868,1.641)$ & \\
\hline \multicolumn{4}{|c|}{ Age at marriage (years) } & \multirow{4}{*}{-} \\
\hline$<18$ & $27.6(25.6,29.6)$ & $25.2(22.7,27.7)$ & Reference*** & \\
\hline 18 to 20 & $54.1(51.8,56.3)$ & $50.5(47.6,53.4)$ & $1.023(0.857,1.220)$ & \\
\hline$\geq 21$ & $18.3(16.6,20.1)$ & $24.3(21.8,26.8)$ & $1.451(1.172,1.796) * * *$ & \\
\hline \multicolumn{5}{|c|}{ Age when first child born (years) } \\
\hline$<21$ & $51.8(49.6,54.1)$ & $45.6(42.7,48.5)$ & Reference & Reference \\
\hline$\geq 21$ & $48.2(45.9,50.4)$ & $54.4(51.5,57.3)$ & $1.286(1.110,1.490) * * *$ & $1.128(0.960,1.324)$ \\
\hline \multicolumn{4}{|l|}{ Religion } & \multirow[t]{4}{*}{ (2) } \\
\hline Hindu/Sikh & $84.3(82.7,85.9)$ & $84.1(81.9,86.2)$ & Reference & \\
\hline Muslim & $12.7(11.3,14.2)$ & $12.6(10.7,14.5)$ & $0.991(0.794,1.238)$ & \\
\hline Christian & $2.9(2.2,3.7)$ & $3.3(2.3,4.4)$ & $1.132(0.743,1.725)$ & \\
\hline \multicolumn{5}{|l|}{ Social group } \\
\hline General & $8.5(7.3,9.8)$ & $10.3(8.5,12.1)$ & Reference*** & Reference $* * *$ \\
\hline $\mathrm{SC}$ & $26.8(24.8,28.7)$ & $28.5(25.9,31.2)$ & $0.884(0.671,1.164)$ & $0.997(0.745,1.334)$ \\
\hline ST & $9.7(8.3,11.0)$ & $5.5(4.2,6.9)$ & $0.476(0.328,0.691) * * *$ & $0.542(0.366,0.803) * * *$ \\
\hline $\mathrm{OBC}$ & $55.0(52.8,57.2)$ & $55.7(52.8,58.6)$ & $0.840(0.649,1.086)$ & $0.876(0.670,1.145)$ \\
\hline \multicolumn{5}{|l|}{ Education } \\
\hline Illiterate & $37.1(34.9,39.2)$ & $24.2(21.7,26.7)$ & Reference*** & Reference $* * *$ \\
\hline Primary & $7.4(6.2,8.6)$ & $6.8(5.3,8.3)$ & $1.404(1.029,1.916) * *$ & $1.417(1.029,1.951) * *$ \\
\hline Secondary & $42.3(40.1,44.5)$ & $51.0(48.1,54.0)$ & $1.846(1.548,2.201) * * *$ & $1.668(1.365,2.039) * * *$ \\
\hline Graduate and above & $13.3(11.8,14.8)$ & $18.0(15.7,20.2)$ & $2.073(1.645,2.612) * * *$ & $1.502(1.136,1.986) * * *$ \\
\hline \multicolumn{5}{|l|}{ Occupation } \\
\hline working women & $28.2(26.2,30.2)$ & $28.1(25.4,30.7)$ & Reference & Reference \\
\hline House wife/ Student & $71.8(69.8,73.8)$ & $71.9(69.3,74.6)$ & $1.007(0.855,1.187)$ & $0.675(0.558,0.816) * * *$ \\
\hline \multicolumn{5}{|l|}{ Family size } \\
\hline$<5$ members & $38.3(36.2,40.5)$ & $36.1(33.3,38.9)$ & Reference & Reference \\
\hline$\geq 5$ members & $61.7(59.5,63.8)$ & $63.9(61.1,66.7)$ & $1.100(0.945,1.281)$ & $1.154(0.984,1.353)^{*}$ \\
\hline \multicolumn{5}{|l|}{ Wealth quintile } \\
\hline Lower Class & $10.2(8.8,11.5)$ & $15.7(13.6,17.9)$ & Reference*** & Reference \\
\hline Lower Middle Class & $23.1(21.2,24.9)$ & $28.2(25.6,30.9)$ & $0.792(0.617,1.015) *$ & $0.841(0.648,1.090)$ \\
\hline Middle Class & $26.3(24.4,28.3)$ & $24.6(22.1,27.1)$ & $0.604(0.470,0.776) * * *$ & $0.689(0.521,0.912)^{* * *}$ \\
\hline Upper Middle Class & $22.8(20.9,24.6)$ & $18.9(16.6,21.2)$ & $0.538(0.415,0.699) * * *$ & $0.730(0.531,1.003)^{*}$ \\
\hline Upper Class & $17.7(16.0,19.4)$ & $12.5(10.6,14.4)$ & $0.458(0.345,0.607) * * *$ & $0.671(0.471,0.956) * *$ \\
\hline \multicolumn{5}{|l|}{ Parity } \\
\hline Primipara & $63.5(61.4,65.6)$ & $63.7(60.9,66.5)$ & $0.991(0.851,1.155)$ & \multirow[t]{2}{*}{-} \\
\hline Multipara & $36.5(34.3,38.6)$ & $36.3(33.5,39.1)$ & Reference & \\
\hline \multicolumn{5}{|l|}{ Location } \\
\hline Rural & $52.8(50.6,55.0)$ & $42.9(40.0,45.8)$ & Reference & Reference \\
\hline Urban & $47.2(45.0,49.4)$ & $57.1(54.2,60.0)$ & $1.491(1.286,1.729) * * *$ & $1.342(1.112,1.620) * * *$ \\
\hline \multicolumn{5}{|l|}{ Region } \\
\hline West & $33.9(31.8,36.0)$ & $22.9(20.4,25.3)$ & Reference*** & Reference*** \\
\hline North & $27.0(25.0,29.0)$ & $25.0(22.5,27.6)$ & $1.375(1.121,1.687)^{* * *}$ & $1.570(1.268,1.945) * * *$ \\
\hline East & $20.6(18.8,22.4)$ & $31.5(28.7,34.2)$ & $2.263(1.847,2.773) * * *$ & $2.424(1.966,2.990) * * *$ \\
\hline South & $18.5(16.8,20.2)$ & $20.6(18.3,23.0)$ & $1.654(1.328,2.060)^{* * *}$ & $1.771(1.414,2.219) * * *$ \\
\hline Constant & & & & $0.376^{* * *}$ \\
\hline
\end{tabular}


Table 4: Components of delivery care against adequacy status of ANC in each region of Telangana.

\begin{tabular}{|c|c|c|c|c|c|c|c|c|}
\hline & \multicolumn{2}{|l|}{$\begin{array}{l}\text { North\# } \\
(\mathbf{n}=\mathbf{8 0 5})\end{array}$} & \multicolumn{2}{|l|}{$\begin{array}{l}\text { East\# } \\
(\mathrm{n}=753)\end{array}$} & \multicolumn{2}{|l|}{$\begin{array}{l}\text { South\# } \\
(\mathrm{n}=591)\end{array}$} & \multicolumn{2}{|l|}{$\begin{array}{l}\text { West\# } \\
(n=916)\end{array}$} \\
\hline & $\begin{array}{l}\text { Inadequate } \\
\text { care } \\
(n=525)\end{array}$ & $\begin{array}{l}\text { Adequate } \\
\text { care* } \\
(\mathbf{n}=\mathbf{2 8 0})\end{array}$ & $\begin{array}{l}\text { Inadequate } \\
\text { care } \\
(n=401)\end{array}$ & $\begin{array}{l}\text { Adequate } \\
\text { care* } \\
(\mathbf{n}=\mathbf{3 5 2})\end{array}$ & $\begin{array}{l}\text { Inadequate } \\
\text { care } \\
(\mathbf{n}=\mathbf{3 6 0})\end{array}$ & $\begin{array}{l}\text { Adequate } \\
\text { care* } \\
(\mathbf{n}=\mathbf{2 3 1})\end{array}$ & $\begin{array}{l}\text { Inadequate } \\
\text { care } \\
(n=660)\end{array}$ & $\begin{array}{l}\text { Adequate } \\
\text { care* } \\
(\mathbf{n}=256)\end{array}$ \\
\hline \multicolumn{9}{|l|}{ Type of delivery } \\
\hline Normal & $310(59.0)$ & $82(29.3)$ & $201(50.1)$ & $129(36.6)$ & $192(53.3)$ & $98(42.4)$ & $356(53.9)$ & $83(32.4)$ \\
\hline Caesarean & $215(41.0)$ & $198(70.7)$ & $200(49.9)$ & $223(63.4)$ & $168(46.7)$ & $133(57.6)$ & $304(46.1)$ & $173(67.6)$ \\
\hline \multicolumn{9}{|l|}{ Place of delivery } \\
\hline Govt. Hospital/AYUSH & $212(40.4)$ & $54(19.3)$ & $131(32.7)$ & $90(25.6)$ & $98(27.2)$ & $81(35.1)$ & $203(30.8)$ & $55(21.5)$ \\
\hline Pvt. Hospital/AYUSH & $245(46.7)$ & $213(76.1)$ & $251(62.6)$ & $253(71.9)$ & $231(64.2)$ & $144(62.3)$ & $422(63.9)$ & $193(75.4)$ \\
\hline Home & $68(13.0)$ & $13(4.6)$ & $19(4.7)$ & $9(2.6)$ & $31(8.6)$ & $6(2.6)$ & $35(5.3)$ & $8(3.1)$ \\
\hline \multirow[b]{2}{*}{$\begin{array}{l}\mathrm{n}=\text { number of eligible } \\
\text { women for JSY scheme. }\end{array}$} & $(n=737)$ & & $(n=635)$ & & $(n=531)$ & & $(\mathrm{n}=778)$ & \\
\hline & $\begin{array}{l}\text { Inadequate } \\
\text { care } \\
(\mathrm{n}=492)\end{array}$ & $\begin{array}{l}\text { Adequate } \\
\text { care* } \\
(\mathrm{n}=245)\end{array}$ & $\begin{array}{l}\text { Inadequate } \\
\text { care } \\
(\mathrm{n}=342)\end{array}$ & $\begin{array}{l}\text { Adequate } \\
\text { care* } \\
(\mathrm{n}=293)\end{array}$ & $\begin{array}{l}\text { Inadequate } \\
\text { care } \\
(\mathrm{n}=328)\end{array}$ & $\begin{array}{l}\text { Adequate } \\
\text { care* } \\
(n=203)\end{array}$ & $\begin{array}{l}\text { Inadequate } \\
\text { care } \\
(\mathrm{n}=564)\end{array}$ & $\begin{array}{l}\text { Adequate } \\
\text { care* } \\
(\mathrm{n}=214)\end{array}$ \\
\hline Received JSY & $\begin{array}{l}90 \\
(18.3 \%)\end{array}$ & $25(10.2 \%)$ & $\begin{array}{l}72 \\
(21.1 \%)\end{array}$ & $75(25.6 \%)$ & $\begin{array}{l}61 \\
(18.6 \%)\end{array}$ & $56(27.6 \%)$ & $88(15.6 \%)$ & $29(13.6 \%)$ \\
\hline \multirow[b]{2}{*}{$\begin{array}{l}\mathrm{n}=\text { number of } \\
\text { Institutional deliveries. }\end{array}$} & $(\mathrm{n}=724)$ & & $(n=725)$ & & $(\mathrm{n}=554)$ & & $(\mathrm{n}=873)$ & \\
\hline & $\begin{array}{l}\text { Inadequate } \\
\text { care } \\
(\mathrm{n}=457)\end{array}$ & $\begin{array}{l}\text { Adequate } \\
\text { care* } \\
(\mathrm{n}=267)\end{array}$ & $\begin{array}{l}\text { Inadequate } \\
\text { care } \\
(\mathrm{n}=382)\end{array}$ & $\begin{array}{l}\text { Adequate } \\
\text { care* } \\
(\mathrm{n}=343)\end{array}$ & $\begin{array}{l}\text { Inadequate } \\
\text { care } \\
(\mathrm{n}=329)\end{array}$ & $\begin{array}{l}\text { Adequate } \\
\text { care* } \\
(\mathrm{n}=225)\end{array}$ & $\begin{array}{l}\text { Inadequate } \\
\text { Care } \\
(\mathrm{n}=625)\end{array}$ & $\begin{array}{l}\text { Adequate } \\
\text { care* } \\
(\mathrm{n}=248)\end{array}$ \\
\hline \multicolumn{9}{|l|}{$\begin{array}{l}\text { Transport } \\
\text { arrangement }\end{array}$} \\
\hline Doctor & $27(5.9)$ & $3(1.1)$ & $26(6.8)$ & $6(1.7)$ & $19(5.8)$ & $4(1.8)$ & $36(5.8)$ & $8(3.2)$ \\
\hline ANM/HW/AWW/ASHA & $41(9.0)$ & $3(1.1)$ & $21(5.5)$ & $0(0.0)$ & $10(3.0)$ & $12(5.3)$ & $37(5.9)$ & $4(1.6)$ \\
\hline Husband & $249(54.5)$ & $145(54.3)$ & $228(59.7)$ & $234(68.2)$ & $226(68.7)$ & $159(70.7)$ & $429(68.6)$ & $178(71.8)$ \\
\hline Family/Relatives/Friends & $121(26.5)$ & $114(42.7)$ & $103(27.0)$ & $99(28.9)$ & $65(19.8)$ & $46(20.4)$ & $114(18.2)$ & $56(22.6)$ \\
\hline Self & $19(4.2)$ & $2(0.7)$ & $4(1.0)$ & $4(1.2)$ & $9(2.7)$ & $4(1.8)$ & $9(1.4)$ & $2(0.8)$ \\
\hline \multicolumn{9}{|l|}{ Mode of transport } \\
\hline Ambulance & $29(6.3)$ & $8(3.0)$ & $25(6.5)$ & $19(5.5)$ & $13(4.0)$ & $22(9.8)$ & $78(12.5)$ & $13(5.2)$ \\
\hline Personal transport & $392(85.8)$ & $255(95.5)$ & $335(87.7)$ & $309(90.1)$ & $286(86.9)$ & $200(88.9)$ & $483(77.3)$ & $207(83.5)$ \\
\hline Public transport & $36(7.9)$ & $4(1.5)$ & $22(5.8)$ & $15(4.4)$ & $30(9.1)$ & $3(1.3)$ & $64(10.2)$ & $28(11.3)$ \\
\hline
\end{tabular}

\section{Measures}

Outcome variable: The outcome or dependent variable is adequacy of ANC received by mothers:

- Adequate care is defined as mothers receiving at least 4 ANC visits, at least 1 Tetanus Toxoid (TT) injection and consumption of 100 or more iron-folic acid (IFA) tablets.

- Inadequate care is defined as mothers receiving less than 4 ANC visits, no TT injection and consumption of less than 100 IFA tablets.

Explanatory variable: Socio-demographic variables were mother's age in years, age at marriage in years, mother's age at first child birth in years, religion, social group, educational status, occupational status, family size, wealth quintile, parity, location, geographical region of Telangana state (Telangana state stratified into 4 regions).

\section{Statistical analysis}

The analysis was carried out using IBM SPSS Statistics 20.0 (IBM Corp., Armonk, NY, USA). The proportions with $95 \%$ confidence intervals (CI) for all the components of antenatal care and access to logistic services at public health facilities for intra-partum care were reported stratified by regions of Telangana state and adequacy of ANC.

The proportions with $95 \%$ CI were also carried out for all components of postnatal care stratified by regions of Telangana. Univariate logistic regression was carried out to determine the assumption of independence of determinants of antenatal care prior to binomial logistic regression for adequate ANC. Backward likelihood ratio method was carried out to find the predictors of adequacy of ANC with maximum P-value of 0.25 and HosmerLemeshow goodness of fit was carried out for the model fitting. A stacked bar chart was used to report the adequacy of ANC within the districts of Telangana. 


\section{RESULTS}

\section{Regional variation in maternal health services utilization}

Table 1 reveals mothers with higher secondary education had more likely availed antenatal services among four regions of the state of Telangana compared to other groups. The majority of mothers availing antenatal services were housewives among all regions. Mothers of middle class had more likely availed antenatal services in East and South regions while upper and lower middle class in North and West regions, respectively. Majority of prospective mothers availing antenatal services were married between 18-20 years of age across all regions.
Table 2 shows mother's receiving adequate ANC were highest in East region (46.7\%) and lowest in West region $(27.9 \%)$. Private hospitals are main source of provision of antenatal care services in all regions of Telangana. At least 74 to $82 \%$ of mothers had registered for ANC services in the first trimester across all regions of Telangana. Consumption of at least 100 iron-folic acid tablets by pregnant mothers is $53.1 \%$ and $50.9 \%$ in East and South regions of Telangana, while $42.1 \%$ and $35.4 \%$ in North and West regions of Telangana, respectively.

Pregnant women reception of two doses of tetanus vaccines is highest in East $(63.2 \%)$ and lowest in West $(50.0 \%)$ regions.

Table 5: Components of post-natal care against the regions of Telangana.

\begin{tabular}{|c|c|c|c|c|}
\hline \multirow[b]{2}{*}{ Components of post-natal care } & \multicolumn{4}{|l|}{ Regions } \\
\hline & $\begin{array}{l}\text { North }{ }^{\#} \\
(n=804)\end{array}$ & $\begin{array}{l}\text { East }^{\#} \\
(n=742)\end{array}$ & $\begin{array}{l}\text { South }^{\#} \\
(n=585)\end{array}$ & $\begin{array}{l}\text { West }^{\#} \\
(n=911)\end{array}$ \\
\hline $\begin{array}{l}\text { Maternal check-up done within } \\
\text { 48hours }\end{array}$ & $73.9(70.8,76.9)$ & $78.2(75.2,81.1)$ & $74.9(71.4,78.4)$ & $64.4(61.3,67.5)$ \\
\hline \multicolumn{5}{|l|}{ Natal check-up } \\
\hline Within 24 hours & $60.2(56.8,63.6)$ & $61.9(58.4,65.4)$ & $57.1(53.1,61.1)$ & $49.9(46.7,53.2)$ \\
\hline 24 to 72 hours & $18.3(15.6,21.0)$ & $25.5(22.3,28.6)$ & $28.4(24.7,32.0)$ & $27.4(24.5,30.3)$ \\
\hline After 72 hours & $9.5(7.4,11.5)$ & $3.4(2.1,4.7)$ & $3.4(1.9,4.9)$ & $5.7(4.2,7.2)$ \\
\hline Colostrum given & $96.6(95.4,97.9)$ & $95.8(94.4,97.3)$ & $96.9(95.5,98.3)$ & $94.0(92.4,95.5)$ \\
\hline \multicolumn{5}{|l|}{ Time of initiation of breast milk } \\
\hline Within an hour & $56.2(52.8,59.6)$ & $48.2(44.7,51.8)$ & $55.7(51.7,59.8)$ & $55.5(52.3,58.8)$ \\
\hline Within 24 hours & $90.2(88.1,92.2)$ & $89.4(87.1,91.6)$ & $90.6(88.2,93.0)$ & $85.7(83.5,88.0)$ \\
\hline \multirow[t]{2}{*}{ After 24 hours } & $9.1(7.1,11.1)$ & $9.7(7.6,11.8)$ & $8.5(6.3,10.8)$ & $10.9(8.8,12.9)$ \\
\hline & $(n=713)$ & $(n=686)$ & $(n=533)$ & $(n=840)$ \\
\hline \multirow[t]{2}{*}{ Low birth weight } & $13.9(11.3,16.4)$ & $25.4(22.1,28.6)$ & $15.9(12.8,19.1)$ & $15.1(12.7,17.6)$ \\
\hline & $(n=704)$ & $(\mathrm{n}=639)$ & $(n=504)$ & $(\mathrm{n}=802)$ \\
\hline Exclusive breastfeeding & $54.5(50.8,58.2)$ & $54.8(50.9,58.7)$ & $58.7(54.4,63.0)$ & $61.2(57.8,64.6)$ \\
\hline
\end{tabular}

Mahabubnagar, Nalgonda; West = Medak, Hyderabad, Rangareddy .

Table 3 illustrates $6.8 \%$ illiterates, $24.2 \%$ prospective pregnant mothers with primary education, $51 \%$ with secondary education and $18 \%$ with graduation and above had received adequate ANC services. Reception of adequate ANC was high (71.9\%) in house wives or students compared to working women.

Adequacy of ANC was high (28.2\%) in pregnant mothers belongs to lower middle class compared to others and is high in urban region $(57.1 \%)$. pregnant women of East region were 2.4 times more likely to receive antenatal services compared to West and higher proportion (31\%) compared to all other regions.

Table 4 elucidates higher institutional deliveries took place in private hospitals in all regions of Telangana. Janani Suraksha Yojana (JSY) program utilization was higher in East $(25.6 \%)$ and South $(27.6 \%)$ regions compared to North $(10.2 \%)$ and West $(13.6 \%)$ regions.

Table 5 reveals postnatal services provided in Telangana. The status of maternal check-up done within 48 hours was high in East region $(78.2 \%)$ followed by South $(74.9 \%)$ and North $(73.9 \%)$ regions and is low in West region $64.4 \%$ ). Highest post-natal check-ups within 24 hours was recorded in East region $(61.9 \%)$, followed by North $(60.2 \%)$ and South $(57.1 \%)$ and was lowest in West $(49.9 \%)$ region. Time of initiation of breast milk within an hour was high in North (56.2\%), South (55.7\%) and West regions $(55.5 \%)$ and was low in East $(48.2 \%)$ region. The percentage of children born with low birth weight was high in East region (25.4\%) compared to South (15.9\%), West (15.1\%) and North (13.9\%) regions. Exclusive breast feeding was high in West region 
(61.2\%) compared to South (58.7\%), East (54.8\%) and North $(54.5 \%)$ regions.

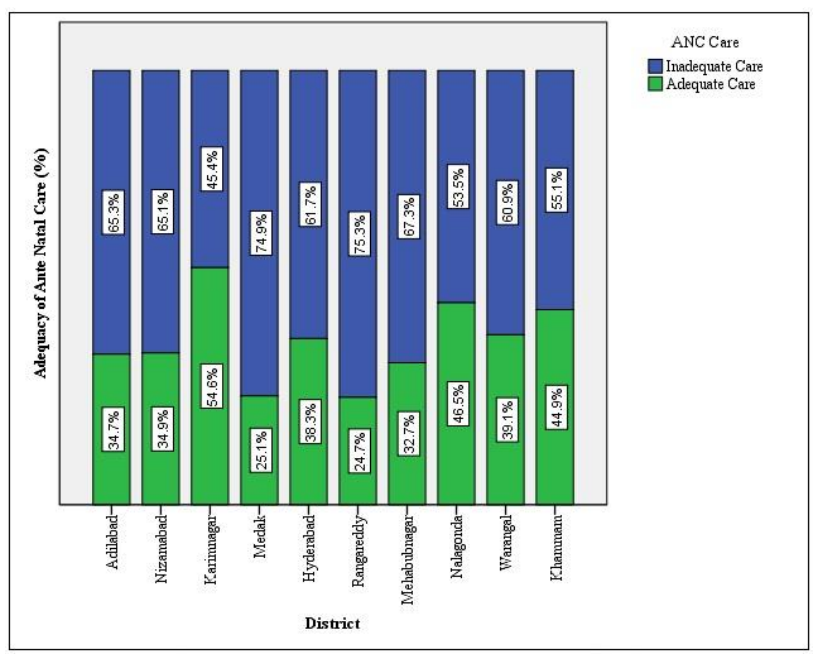

Figure 1: Utilization of antenatal services by pregnant women stratified by districts in Telangana.

Figure 1 reveals adequacy of utilization of antenatal services stratified by erstwhile district of Telangana state. Pregnant women of Karimnagar district were foremost in utilization of services compared to other districts while pregnant women of Medak and Rangareddy were poor in utilization.

\section{DISCUSSION}

Utilization of adequate ANC services is $42.2 \%$ in the Telangana state compared to $32.9 \%, 43.7 \%$, and $45 \%$ in Karnataka, Andhra Pradesh, and Tamil Nadu, respectively. ${ }^{20}$ Wide variation in utilization of ANC services across different districts of Telangana with the reception of adequate care is low in Ranga Reddy (24.7\%) and Medak (25.1\%) district and high in Karimnagar district (54.6\%). Therefore, variation of uptake of reception of ANC services across districts is due to demand and supply side barriers of access to maternal health services in communities. ${ }^{21,22}$

Immediate breast feeding and breast feeding within onehour practice to newborn is effective in prevention of post-partum haemorrhage of pregnant women. ${ }^{23}$ Our data reveals practice of breast feeding within one hour is $57.2 \%$ and this practice and of immediate breast feeding should be implemented effectively in the facilities and communities to improve maternal outcomes.

Supply-side barriers comprise poor attitude of health care staff of public health facilities toward communities at the lower end of wealth quintile. Lack of medicines at the health center had become a major share of out of pocket expenses for poor HHs hindering them to avail maternal health services. ${ }^{24}$ Non-availability of health providers at public health facilities is the frequent complaint received from the communities and forcing them to utilize the private health facilities increasing the cost of care and a major economic burden to them. ${ }^{25}$ Utilization of private health facilities for ANC services is high across all regions of Telangana.

Demand-side barriers override supply side and are a major cause of inadequate utilization of maternal health services. ${ }^{23}$ Firstly, indirect consumer costs such as transportation to health facility is a major barrier for availing ANC services and institutional deliveries. Transport costs to distant health facility is a major barrier affecting the utilization of services. ${ }^{22,24}$ Secondly, financial constraint is one of the major reasons for home deliveries, hence government of India launched JSY scheme on April 12, 2015, aimed to reduce neonatal and maternal mortality rates by promoting institutional delivery. ${ }^{22,26,27}$

JSY scheme utilization is $14.3 \%, 23.3 \%, 23.1 \%$, and $14.6 \%$ in North, East, South and West region, respectively and is inversely proportional to institutional deliveries at private hospitals. Low utilization of the scheme might be due to lack of awareness of scheme and no motivation from health workers in utilizing it. Thirdly, lack of community engagement in seeking health-care services, institutional deliveries, and perinatal care is one of the barriers in utilizing services. Evidence from previous intervention studies reveal Lady Health Workers (LHW) are effective in promoting maternal health services by various approaches, for example, home visits, home management, and facilitated referral.

Home visits involve provision of basic ANC (nutrition counseling, screening for common illness, iron-folic acid, and TT administration), newborn care preparedness and home-based perinatal care by LHW. ${ }^{28}$ Another approach like LHW organizing group sessions in the community to promote ANC, use of clean kits at delivery, institutional delivery, identifying danger signs of pregnancy, and promotion of health seeking behavior. ${ }^{29}$ Evidence from previous studies shows recruitment of ASHA health worker in rural areas had increased the seeking of maternal health services (registration in the first trimester, ANC visits, institutional deliveries, deliveries by skilled birth attendant, immunization). ${ }^{30}$

The strategies designed for engaging the community are not meeting the targets due to lack of knowledge of community about strategies designed without understanding three fundamental influences of decisionmaking process for the woman making health-care decision. These influences are gendered decision-making norms, multigenerational dialogue, and appropriate communication.

Gendered decision-making norms play a vital role in influencing health-seeking behavior and health outcomes. Gender equality and women empowerment are essential to increase utilization of maternal health services. At the $\mathrm{HH}$ level disempowerment of women, results to lowered 
access to resources such as education, employment, and income, and limits their decision-making power. ${ }^{24}$ Factors increasing women role in decision-making process in a $\mathrm{HH}$ is their education and contribution to $\mathrm{HH}$ income. Our study corroborates these findings that women with secondary education had a higher probability of adequate ANC and institutional delivery. The difference in education between wife and husband is also crucial in influencing decision-making process. ${ }^{31}$

Multigenerational gaps exist as social norms, values, traditions, and customs inhibit mothers to transfer knowledge and experiences to their daughters resulting in lack of sexual education and preparedness for motherhood. ${ }^{32,33}$ Generational gaps are growing reality due to increased urbanization and proliferation of nuclear families leading to increasing disconnection of urban HHs from their traditional roots, conflict between social beliefs can arise.

Intergenerational dialogue is necessary to achieve consensus on best practices in motherhood. The lack of communication between pregnant women and health provider leads to poor health outcomes. ANC can be delivered by frontline providers including midwives, nurses, and community health workers, provided they are well trained. Health information should be designed in consultation with local women and disseminated in twoway communication considering literacy levels of mothers.

A limitation of DLHS-4 survey is that limited data were collected of supply side factors at village level about the availability of health-care infrastructure, but no information was collected about barriers to accessibility and quality of maternal health services from HHs. Another limitation of the study is retrospective reporting, which involves recall bias and therefore, impacts the reliability of data.

\section{CONCLUSION}

Wide variation of utilization of maternal health services across four regions of Telangana because of lack of awareness of mothers of various health schemes, financial constraints, and lack of awareness of HHs of incentives for institutional delivery. Health interventions should be designed to tackle the barriers such as effective training of ASHA worker and nurses to educate and motivate mothers about newborn and implementation of breast feeding practices in facilities and evidence based practices of intra-partum and post-partum care and, engaging communities for women empowerment, identification of community volunteers to motivate women to seek health care. Policy measures to be adopted to periodically monitor the supply side issues like the application of Geographical Information System to assess the accessibility of maternal health services in underperforming districts, and improving infrastructure, and quality of care of public health facilities.

\section{ACKNOWLEDGMENTS}

Authors would like to thank IIPS, Mumbai for providing DLHS-4 data.

Funding: No funding sources

Conflict of interest: None declared

Ethical approval: The study was approved by the Institutional Ethics Committee

\section{REFERENCES}

1. Jat TR, Ng N, San Sebastian M. Factors affecting the use of maternal health services in Madhya Pradesh state of India: a multilevel analysis. Internat J Equity Health. 2011;10(1):59.

2. World Health Organization. Health in 2015 from MDGs to SDGs. Geneva: World Health Organization; 2015.

3. Planning Commission. High level expert group report on universal health coverage for India; 2011.

4. Kesterton AJ, Cleland J, Sloggett A, Ronsmans C. Institutional delivery in rural India: the relative importance of accessibility and economic status. BMC Pregnancy aChildbirth. 2010;10(1):30.

5. International Institute for Population Sciences. India National Family Health Survey (NFHS-3), 2005-06. International Institute for Population Sciences; 2007.

6. Pathak PK, Singh A, Subramanian SV. Economic inequalities in maternal health care: prenatal care and skilled birth attendance in India, 1992-2006. PloS one. 2010;5(10):e13593.

7. Singh PK, Rai RK, Kumar C. Equity in maternal, newborn, and child health care coverage in India. Global health action. 2013;6(1):22217.

8. Kumar A, Mohanty SK. State of child health among poor and non-poor in urban India. Genus. 2011;67(1):1-9.

9. Bonu S, Bhushan I, Rani M, Anderson I. Incidence and correlates of 'catastrophic'maternal health care expenditure in India. Health Policy Plann. 2009;24(6):445-56.

10. Skordis-Worrall J, Pace N, Bapat U, Das S, More NS, Joshi W, et al. Maternal and neonatal health expenditure in Mumbai slums (India): a cross sectional study. BMC Public Health. 2011;11(1):150.

11. Lincetto $\mathrm{O}$, Mothebesoane-Anoh S, Gomez $\mathrm{P}$, Munjanja S. Antenatal Care: Opportunities for Africa's Newborns. New York: World Health Organiation; 2010.

12. Requejo JH, Bryce J, Victora CG. Building a future for women and children: the 2012 report. World Health Organization; 2012.

13. Campbell OM, Graham WJ, Lancet Maternal Survival Series steering group. Strategies for reducing maternal mortality: getting on with what works. Lancet. 2006;368(9543):1284-99.

14. Villar J, Ba'aqeel H, Piaggio G, Lumbiganon P, BelizánJM, Farnot U, et al. WHO antenatal care randomised trial for the evaluation of a new model of 
routine antenatal care. Lancet. 2001;357(9268):155164.

15. World Health Organization. WHO antenatal care randomized trial: manual for the implementation of the new model. Geneva: World Health Organization. 2002:37.

16. World Health Organization, UNICEF. Antenatal care in developing countries: Promises, Achievements and Missed opportunities: An analysis of trends, levels, and differentials: 1990-2001. Geneva, New York: WHO and UNICEF. 2003.

17. Houweling TA, Ronsmans C, Campbell OM, Kunst AE. Huge poor-rich inequalities in maternity care: an international comparative study of maternity and child care in developing countries. Bulletin of the World Health Organization. 2007;85(10):745-54.

18. Simkhada B, Teijlingen ER, Porter M, Simkhada P. Factors affecting the utilization of antenatal care in developing countries: systematic review of the literature. J Advanced Nurs. 2008;61(3):244-60.

19. Ministry of Statistics and Programme Implementation. Statistical Year Book 2016. Stastical Year B. 2016;(42). Available at http://www.mospi.gov.in/statistical-year-bookindia/2016.

20. International Institute for Population Sciences (IIPS) and Macro International. National Family Health Survey (NFHS-4), India, 2015-16: India. Mumbai: IIPS; 2015.

21. Ensor T, Cooper S. Overcoming barriers to health service access: Influencing the demand side. Health Policy Plan. 2004;19:69-79.

22. Elmusharaf K, Byrne E, O'Donovan D. Strategies to increase demand for maternal health services in resource-limited settings: Challenges to be addressed. BMC Public Health. 2015;15:870.

23. Matthiesen A-S, Ransjö-Arvidson A-B, Nissen E, Uvnäs-Moberg K. Postpartum Maternal Oxytocin Release by Newborns: Effects of Infant Hand Massage and Sucking. Birth. 2001;28(1):13-9.

24. Piet-Pelon NJ, Rob U, Khan ME. Men in Bangladesh, India and Pakistan: Reproductive Health Issues. Dhaka: Karshaf Publishers; 1999:184.

25. Gender Equality and Women's Empowerment in India, National Family Health Survey 3, 2005-2006.
International Institute of Population Sciences; 20052006.

26. Geddam JB, Ponna SN, Kommu PR, Kokku SB, Mamidi S, Bontha VB. Utilization of maternal health services by the migrant population living in the nonnotified slums of Hyderabad city, India. Indian $\mathbf{J}$ Comm Health. 2017;29(1):29-38.

27. Janani Suraksha Yojana (JSY). Available from: http://www.nhp.gov.in/janani-suraksha-yojana-jsy-pg

28. Baqui AH, El-Arifeen S, Darmstadt GL, Ahmed S, Williams EK, Seraji HR, et al. Effect of communitybased newborn-care intervention package implemented through two service-delivery strategies in Sylhet district, Bangladesh: A cluster-randomised controlled trial. Lancet. 2008;371:1936-44.

29. Bhutta ZA, Soofi S, Cousens S, Mohammad S, Memon ZA, Ali I, et al. Improvement of perinatal and newborn care in rural Pakistan through community-based strategies: A cluster-randomised effectiveness trial. Lancet. 2011;377:403-12.

30. Padda P, Devgun S, Gupta V, Chaudhari S, Singh G. Role of ASHA in improvement of maternal health status in northern India: An urban rural comparison. Indian J Community Health. 2013;25:465-71.

31. Quisumbing AR, Maluccio JA. Intrahousehold Allocation and Gender Relations: New Empirical Evidence from Four Developing Countries. Washington, DC: International Food Policy Research Institute; 2000.

32. Varga CA. How gender roles influence sexual and reproductive health among South African adolescents. Stud Fam Plann. 2003;34:160-72.

33. Hutchinson MK, Jemmott JB, Jemmott LS, Braverman P, Fong GT. The role of mother-daughter sexual risk communication in reducing sexual risk behaviors among urban adolescent females: A prospective study. J Adolesc Health 2003;33:98-107.

Cite this article as: Singh N, Ponna SN, Upadrasta VP, Dudala SR, Sadasivuni R. Determinants of utilization of antenatal and postnatal care services in Telangana. Int J Reprod Contracept Obstet Gynecol 2017;6:3352-61. 